\title{
MOLECULAR TRACERS OF PDR-DOMINATED GALAXIES
}

\author{
E. Bayet ${ }^{1}$, S. Viti ${ }^{1}$, D. A. Williams ${ }^{1}$, J. M. C. Rawlings ${ }^{1}$, And T. Bell ${ }^{2}$ \\ ${ }^{1}$ Department of Physics and Astronomy, University College London, Gower Street, London WC1E 6BT, UK; eb@star.ucl.ac.uk \\ ${ }^{2}$ Department of Astronomy, California Institute of Technology, Pasadena, CA 91125, USA \\ Received 2008 November 12; accepted 2009 February 23; published 2009 April 24
}

\begin{abstract}
Photon-dominated regions (PDRs) are powerful molecular line emitters in external galaxies. They are expected in galaxies with high rates of massive star formation due to either starburst (SB) events or SB coupled with active galactic nuclei (AGNs) events. We have explored the PDR chemistry for a range of physical conditions representing a variety of galaxy types. Our main result is a demonstration of the sensitivity of the chemistry to changes in the physical conditions. We adopt crude estimates of relevant physical parameters for several galaxy types and use our models to predict suitable molecular tracers of those conditions. The set of recommended molecular tracers differs from that which we recommended for use in galaxies with embedded massive stars. Thus, molecular observations can in principle be used to distinguish between excitation by SB and by SB+AGN in distant galaxies. Our recommendations are intended to be useful in preparing Herschel and ALMA proposals to identify sources of excitation in galaxies.
\end{abstract}

Key words: astrochemistry - galaxies: active - ISM: abundances - ISM: molecules - methods: numerical - stars: formation

\section{INTRODUCTION}

Galaxies may be powerful molecular line emitters because of a very high massive star formation rate (SFR) induced either by starburst (SB) events or the presence of active galactic nuclei (AGNs; Radford et al. 1991; Solomon et al. 1992, 2004; Gerin \& Phillips 2001; Israel \& Baas 2001, 2002, 2003; Bayet et al. 2004, 2006; Martín et al. 2005, 2006; Evans et al. 2006; GraciáCarpio et al. 2006; Aalto et al. 2007; Bell et al. 2007; Imanishi et al. 2007; Knudsen et al. 2007; Papadopoulos 2007; Krips et al. 2008). Some external sources may be dominated by only one type of activity or contain strong signatures of both. Both activity types (SB and AGN) are recognized as having a direct influence on the molecular gas emission in a galaxy.

Molecular emission from dense star-forming cores in SB galaxies has recently been the subject of a modeling study (Bayet et al. 2008b). That work predicts that such galaxies should be strong emitters in rotational lines from molecules embedded in the warm cores from which the young massive stars are evolving (see also Lintott et al. 2005; Lintott \& Viti 2006). Bayet et al. (2008b) described how the chemistry in these cores may depend on the local physical conditions, and they identified some molecular species that should be sensitive tracers of these conditions.

Such SB galaxies may also contain giant photon-dominated regions (PDRs) which are also powerful emitters in molecular lines in the millimeter and submillimeter regions of the spectrum. These PDRs are quite distinct physical and chemical systems from those discussed by Bayet et al. (2008b). Giant PDRs should be set up in galaxies with clusters of young massive stars and in galaxies dominated by radiation from an active nucleus. It is therefore important to discuss the chemistry of galaxies dominated by PDRs, set up either by clusters of young massive stars or by the additional presence of AGNs, and to identify the likely molecular tracers of such PDRs. In particular, it would be important to know whether these molecular signatures are similar to or distinct from those of the star-forming cores of SB galaxies described by Bayet et al. (2008b).

In this companion paper to Bayet et al. (2008b), we model the chemistry of PDR-dominated galaxies. As described in the earlier paper, the physical conditions that determine the chemistry in the interstellar medium (ISM) of external galaxies (metallicity and/or relative elemental abundances, dust:gas ratio, dust grain properties, gas density, cosmic ray ionization rate, and far-UV radiation intensity) may differ substantially from those of the Milky Way and for our purposes are regarded as unknown parameters. The chemistry is explored for a wide range of these parameters and its sensitivity to variations in these parameters is studied. We also attempt to assign likely values of these parameters to galaxy type (SB, SB+AGN, and high redshift) and predict suitable molecular tracers for these galaxy types.

The PDR code and the chemical database are described in Section 2, and the physical parameters and their ranges are discussed in Section 3. Results describing the PDR chemistry and its sensitivity to variations in the parameters listed above are presented in Section 4. In Section 5, we make predictions of the detectability of lines of certain potential tracer molecules in PDR-dominated galaxies at low and high redshift, and in Section 6, we give a discussion of our results in relation to observations, to other PDR modeling, and to our earlier warmcore studies, and we summarize our conclusions.

\section{MODEL DESCRIPTION}

PDR models differ from each other in the assumptions adopted, in the geometry, or in the degree of sophistication used for solving the radiative transfer equations, or in the thermal balance and for determining the level population. PDR models also differ from each other by the chemical network adopted and by the consideration (or not) of time dependence. In this paper, we used the time-dependent UCL_PDR code benchmarked in Röllig et al. (2007) and fully described in Bell et al. (2006, 2007).

The UCL_PDR model assumes that a one-dimensional semiinfinite slab is illuminated from one side by FUV photons. The chemistry and thermal balance are calculated self-consistently at each point into the slab and at each time step, producing chemical abundances, emission line strengths, and gas temperatures as a function of depth and time. The UCL_PDR code adopted here 
Table 1

Standard Model Parameters ${ }^{\mathrm{a}}$ (Bell et al. 2006, 2007, and References Therein)

\begin{tabular}{lc}
\hline \hline Grain size & $0.1 \mu \mathrm{m}$ \\
Grain albedo & 0.7 \\
Microturbulence velocity & $1.5 \mathrm{~km} \mathrm{~s}^{-1}$ \\
Mean photon scattering by grain & 0.9 \\
External FUV radiation intensity $\left(=I_{\odot}\right)$ & $1 \mathrm{Habing}^{\mathrm{b}}$ \\
Cosmic ray ionization rate $\left(=\zeta_{\odot}\right)$ & $5.0 \times 10^{-17} \mathrm{~s}^{-1}$ \\
$\mathrm{H}_{2}$ formation rate coefficient $\left(=R_{\odot}\right)$ & $3 \times 10^{-18} \sqrt{T} \exp \left(\frac{-T}{1000}\right) \mathrm{cm}^{3} \mathrm{~s}^{-1}$ \\
Gas:dust mass ratio $\left(=d_{\odot}\right)$ & 100 \\
$\mathrm{Metallicity}\left(=z_{\odot}\right)$ & $\mathrm{Solar}$ values \\
$\mathrm{Si} / \mathrm{H}$ & $8.21 \times 10^{-7}$ \\
$\mathrm{Fe} / \mathrm{H}$ & $3.60 \times 10^{-7}$ \\
$\mathrm{Cl} / \mathrm{H}$ & $1.10 \times 10^{-7}$ \\
$\mathrm{Na} / \mathrm{H}$ & $8.84 \times 10^{-7}$ \\
$\mathrm{Ca} / \mathrm{H}$ & $5.72 \times 10^{-10}$ \\
\hline
\end{tabular}

Notes.

a These parameters are those used in Models 0-12 except if a different value is specified in Table 2 or described in the text.

b The unit of the standard interstellar radiation field (ISRF) intensity is $I_{\odot}=$ $1.6 \times 10^{-3} \mathrm{erg} \mathrm{cm}^{-2} \mathrm{~s}^{-1}$ Habing (1968).

${ }^{\mathrm{c}} z=1=z_{\odot}$ corresponds to solar values of the initial elemental abundance ratios (see Table 3 ), while $z=1 / 10 z_{\odot}$ means that the solar values of the initial elemental abundance ratios have been all divided by the same factor (of 10 in this example).

uses a chemical network containing 131 species and over 1700 reactions from the UMIST99 database (Le Teuff et al. 2000), including ion-molecule, photoionization and photodissociation reactions. The UCL_PDR model also includes some modifications of UMIST99 introduced as part of the PDR benchmarking effort presented in Röllig et al. (2007). The freezeout of atoms and molecules on to grains is neglected in this model. Apart from hydrogen (which is assumed to be initially mainly molecular), the gas is assumed to be initially in a purely atomic form, with initial elemental abundance ratios as free parameters. Initial elemental abundances of all metals are assumed in most cases to scale linearly with the metallicity $\left(z / z_{\odot}\right)$, and the $\mathrm{H}_{2}$ formation rate and the adopted gas-to-dust mass ratio are also assumed to be linear with the metallicity. For a more detailed description of the UCL_PDR model, we refer the reader to Bell et al. (2006, 2007) and references therein.
Table 3

Initial Abundance Ratios Used in Table $2^{\mathrm{a}}$

\begin{tabular}{lcccr}
\hline \hline & ST & CL02 & HW02 & \multicolumn{1}{c}{ UN02 } \\
\hline $\mathrm{C} / \mathrm{H}$ & $1.4 \times 10^{-4}$ & $1.4 \times 10^{-4}$ & $1.4 \times 10^{-4}$ & $1.4 \times 10^{-4}$ \\
$\mathrm{O} / \mathrm{H}$ & $3.2 \times 10^{-4}$ & $4.54 \times 10^{-4}$ & $1.53 \times 10^{-3}$ & $1.18 \times 10^{-3}$ \\
$\mathrm{~N} / \mathrm{H}$ & $6.5 \times 10^{-5}$ & $5.99 \times 10^{-11}$ & $1.58 \times 10^{-9}$ & $3.24 \times 10^{-7}$ \\
$\mathrm{~S} / \mathrm{H}$ & $1.4 \times 10^{-6}$ & $7.59 \times 10^{-6}$ & $1.54 \times 10^{-4}$ & $4.06 \times 10^{-5}$ \\
$\mathrm{He} / \mathrm{H}$ & $7.5 \times 10^{-2}$ & $7.5 \times 10^{-2}$ & $7.5 \times 10^{-2}$ & $7.5 \times 10^{-2}$ \\
$\mathrm{Mg} / \mathrm{H}$ & $5.1 \times 10^{-6}$ & $1.83 \times 10^{-5}$ & $1.2 \times 10^{-4}$ & $6.18 \times 10^{-5}$
\end{tabular}

Note. "The abbreviations "CL02," "HW02," and "UN02" refer to Chieffi \& Limongi (2002), Heger \& Woosley (2002), and Umeda \& Nomoto (2002), respectively (see Section 3). The standard initial elemental abundance ratios ("ST") are from Sembach \& Savage (1996); Sofia et al. (1997); Meyer et al. (1998); Snow et al. (2002); Knauth et al. (2003). Models using the "ST" values of the initial elemental abundance ratios are the models corresponding to $z=z_{\odot}$.

\section{PARAMETER CHOICES}

To perform our study, we used and extended a model grid previously built and presented by Bell et al. (2006, 2007). This grid contains more than 1200 models, each with a different set of input parameters. We restricted our selection to nine models, the most relevant for our study. Table 1 lists the standard (Milky Way) values of the parameters. Table 2 lists the range of parameter values covered by the models we have selected. We adopt solar initial chemical abundances as our standard. Models 9-11 explore the effect of replacing the solar initial elemental abundance ratios by values predicted by early universe models from Chieffi \& Limongi (2002), Heger \& Woosley (2002), and Umeda \& Nomoto (2002) (see Table 3). In these three models, the metallicity, the $\mathrm{H}_{2}$ formation rate, and the gas-to-dust mass ratio are fixed to standard values. As described later in Section 5, Model 12 is intended to represent high-redshift environments.

Models 0-4 investigate the consequences of a reduction of solar metallicity by a factor up to 100 consistently linked with a reduction of the $\mathrm{H}_{2}$ formation rate, of the gas-to-dust ratio, and of the initial elemental abundance ratios. Models 0 and 6 study the consequent changes in the chemistry after an increase of cosmic ray ionization rate from $5 \times 10^{-17} \mathrm{~s}^{-1}$ to $5 \times 10^{-15} \mathrm{~s}^{-1}$. We suggest that the increase of cosmic ray ionization rate may be qualitatively used to simulate XDR-like environments. Finally,

Table 2

Input Parameters of the UCL_PDR Models Used to Perform this Study (see Sections 3 and 4) ${ }^{\mathrm{a}}$

\begin{tabular}{|c|c|c|c|c|c|c|c|c|}
\hline Model & $\begin{array}{c}\text { Metallicity } \\
\left(z_{\odot}\right)\end{array}$ & $\begin{array}{c}\text { Gas-to-Dust } \\
\text { Mass Ratio }\left(d_{\odot}\right)\end{array}$ & $\begin{array}{c}\mathrm{H}_{2} \text { Form. } \\
\text { Rate Coeff. }\left(R_{\odot}\right)\end{array}$ & $A_{v}$ & $\begin{array}{c}\text { Ini. Elem. } \\
\text { Abund. Ratios }\end{array}$ & $\begin{array}{l}\text { FUV Rad. Field } \\
\qquad\left(I_{\odot}\right)\end{array}$ & $\begin{array}{c}\zeta \\
\left(\zeta_{\odot}\right)\end{array}$ & $\begin{array}{c}\text { Gas Density } \\
\left(\mathrm{cm}^{-3}\right)\end{array}$ \\
\hline 0 & 1 & 1 & 1 & 8 & ST & $10^{3}$ & 1 & $10^{4}$ \\
\hline 1 & $1 / 2$ & $1 / 2$ & $1 / 2$ & 8 & $\mathrm{ST} / 2$ & $10^{3}$ & 1 & $10^{4}$ \\
\hline 2 & $1 / 4$ & $1 / 4$ & $1 / 4$ & 8 & $\mathrm{ST} / 4$ & $10^{3}$ & 1 & $10^{4}$ \\
\hline 3 & $1 / 10$ & $1 / 10$ & $1 / 10$ & 8 & $\mathrm{ST} / 10$ & $10^{3}$ & 1 & $10^{4}$ \\
\hline 4 & $1 / 100$ & $1 / 100$ & $1 / 100$ & 8 & $\mathrm{ST} / 100$ & $10^{3}$ & 1 & $10^{4}$ \\
\hline 5 & 1 & 1 & 1 & 3 & ST & $10^{3}$ & 1 & $10^{4}$ \\
\hline 6 & 1 & 1 & 1 & 8 & ST & $10^{3}$ & 100 & $10^{4}$ \\
\hline 7 & 1 & 1 & 1 & 8 & ST & $10^{5}$ & 1 & $10^{4}$ \\
\hline 8 & 1 & 1 & 1 & 8 & ST & $10^{3}$ & 1 & $10^{6}$ \\
\hline 9 & 1 & 1 & 1 & 8 & CL02 & $10^{3}$ & 1 & $10^{4}$ \\
\hline 10 & 1 & 1 & 1 & 8 & HW02 & $10^{3}$ & 1 & $10^{4}$ \\
\hline 11 & 1 & 1 & 1 & 8 & UN02 & $10^{3}$ & 1 & $10^{4}$ \\
\hline 12 & $1 / 10$ & $1 / 10$ & $1 / 10$ & 8 & $\mathrm{ST} / 10$ & $10^{5}$ & 100 & $10^{4}$ \\
\hline
\end{tabular}

Note. "The abbreviation "ST" represents the standard values while the abbreviations "CL02," "HW02," and "UN02" are the initial elemental abundance ratio references detailed in Table 3. This table does not present all the input parameters of the UCL_PDR code for each model but only lists the parameters set to values different from the standard ones. 
a 2 orders of magnitude change in both the FUV radiation field (Models 0 and 7) and the gas density (Models 0 and 8) has been investigated.

Each model provides atomic and molecular abundances as a function of total cloud depth (at visual extinctions of $0<A_{v}<$ 10) for cloud ages of $10^{4}, 10^{5}, 10^{6}, 10^{7}$, and $10^{8} \mathrm{yr}$. For the study of the $A_{v}$ influence (Models 0 and 5 in Table 2), we have restricted our model selection to those showing either a value of $A_{v}=8$, or a value of $A_{v}=3$ (typical of a translucent gas component). Note that Model 0 is considered as our reference model in this paper, assuming standard parameters (listed in Table 1) with an $A_{v}=8$, likely to be a typical value for representing the dense PDR gas component in galaxies, as detected in the nuclei of M 82, NGC 253, IC 342, and NGC 4038 (Bayet et al. 2008a).

\section{SENSITIVITY OF CHEMICAL ABUNDANCES TO VARIATIONS OF THE UCL_PDR MODEL PARAMETERS}

In this section, we present results on the trends in the chemistry with respect to the changes of various parameters. We have selected 19 molecules for closer study, either for their likely detectability or for their chemical interest (or both). This set of 19 molecules is closely similar to the set of molecules used in Bayet et al. (2008b). We use this set to make a comparison between the predictions of the PDR model and those of models describing the chemistry of high-mass star-forming regions (see Section 6). We have arbitrarily fixed the limit of detectability of molecule $\mathrm{X}$ to be a relative abundance of $n(\mathrm{X}) / n_{\mathrm{H}}=1 \times 10^{-12}$ (where $n_{\mathrm{H}}=n(\mathrm{H})+2 n\left(\mathrm{H}_{2}\right)$ ), a criterion roughly satisfied in our own galaxy. The article deals specifically with molecules $\mathrm{CO}$, $\mathrm{H}_{2} \mathrm{O}, \mathrm{CN}, \mathrm{OH}, \mathrm{CS}, \mathrm{HNC}, \mathrm{HCN}, \mathrm{HCO}^{+}, \mathrm{H}_{3} \mathrm{O}^{+}, \mathrm{SO}, \mathrm{C}_{2}$, and $\mathrm{C}_{2} \mathrm{H}$ (see Figures 1 and 2) and with $\mathrm{CO}_{2}$, OCS, $\mathrm{SO}_{2}, \mathrm{H}_{2} \mathrm{~S}, \mathrm{H}_{2} \mathrm{CS}$, $\mathrm{CH}_{2} \mathrm{CO}$, and $\mathrm{H}_{2} \mathrm{CO}$ (see Tables 4-9). Figures 1-6 show the time evolution of molecular abundances and their sensitivity to parameter changes. Unlike for lower density models (Bell et al. 2006 ), the chemical steady state is achieved here in $\approx 10^{5} \mathrm{yr}$. Thus, time dependence is unlikely to play a role in the chemistry.

\subsection{Metallicity}

Figure 1 shows that the model with the lowest metallicity (Model 4 in Table 2) predicts very low abundances for all species except $\mathrm{CO}, \mathrm{H}_{2} \mathrm{O}, \mathrm{HCO}^{+}$, and $\mathrm{OH}$, and enhances significantly the abundance of $\mathrm{H}_{3} \mathrm{O}^{+}$. This enhancement is caused by a severe reduction in the electron density at low metallicity. Since the main loss of polyatomic ions is through dissociative recombination, the ion abundance increases.

With $z / z_{\odot}=0.01$, we expect that the deuterated ions such as $\mathrm{H}_{2} \mathrm{D}^{+}$and $\mathrm{H}_{2} \mathrm{DO}^{+}$may be more readily detectable than $\mathrm{H}_{3} \mathrm{O}^{+}$(see, e.g., Boreiko \& Betz 1993; Pagani et al. 2007, and references therein). The deuteration in both PDR and highmass star forming models will be investigated in a forthcoming paper.

For models having a metallicity equal to or greater than 1/ $10 z_{\odot}$ (Models 0-3 in Table 2), we can separate species into three different categories: (1) species with relative abundances varying linearly with the metallicity changes, e.g., $\mathrm{CO}, \mathrm{H}_{2} \mathrm{O}$, $\mathrm{CS}, \mathrm{SO}$ (see Figure 1) and $\mathrm{CO}_{2}, \mathrm{SO}_{2}, \mathrm{OCS}$, and $\mathrm{H}_{2} \mathrm{~S}$ (see Table 4); (2) molecules such as $\mathrm{CN}, \mathrm{OH}, \mathrm{HCN}$, and $\mathrm{HNC}$ showing chemical abundances that are rather insensitive to the metallicity changes; and (3) species whose abundance changes are inversely dependent on $z$, such as $\mathrm{C}_{2}, \mathrm{C}_{2} \mathrm{H}$ (see Figure 1), and $\mathrm{H}_{2} \mathrm{CO}$, $\mathrm{CH}_{2} \mathrm{CO}$, and $\mathrm{H}_{2} \mathrm{CS}$ (see Table 4).
Such varieties of behavior have been found in the earliest studies of chemical sensitivity to parameters (Pickles \& Williams 1981) and in recent work (Bayet et al. 2008b) and it is reasonably well understood. Category (1) species may be lost to processes independent of metallicity (e.g., $\mathrm{CO}$ is lost to dissociative ionization with $\mathrm{He}^{+}$), while category (2) species are both formed and destroyed in exchange reactions whose reactants depend on metallicity and are therefore largely neutral to metallicity changes (for example, $\mathrm{OH}$ depends mainly on oxygen atoms for its formation and on exchange reactions with $\mathrm{O}$ and other atoms for its loss). Formation schemes for some category (3) species depend on the production of $\mathrm{C}^{+}$ions from $\mathrm{CO}$, a molecule proportional to $z$, but loss by a succession of exchange reactions resulting in a strong inverse dependence on $z$; thus, there is an overall inverse dependence of the abundance of such species on $z$.

\subsection{Initial Elemental Abundance Ratios}

We have investigated the consequences of making changes in the initial elemental abundance ratios using Models 9-11 (see Table 2). These three models correspond to a study of the influence of a decrease of nitrogen (Model 9), and an increase of both the oxygen and sulfur contents (Models 10 and 11). The values of the corresponding initial elemental abundance ratios are listed in Table 3. Model 9 refers to values from Chieffi \& Limongi (2002), while Models 10 and 11 used the values suggested by Heger \& Woosley (2002) and Umeda \& Nomoto (2002), respectively. Several time-dependent chemical trends are shown in Figure 2.

Neither oxygen- and sulfur-rich environments nor nitrogenpoor environments strongly affect the values of the abundances of $\mathrm{CO}, \mathrm{OH}, \mathrm{H}_{2} \mathrm{~S}, \mathrm{HCO}^{+}, \mathrm{H}_{2} \mathrm{O}$, and $\mathrm{H}_{3} \mathrm{O}^{+}$. Indeed, after $10^{5} \mathrm{yr}$, the scatter in their relative abundances is small, within a factor of $\leqslant 10$. The same behavior is seen for the $\mathrm{CO}_{2}$ relative abundance. These species are thus likely to be irrelevant as tracers of initial metal gas content. On the contrary, SO and CS (see Figure 2) as well as OCS and $\mathrm{SO}_{2}$ abundances (see Table 5) are maximum for the oxygen- and sulfur-rich models and the variation of their relative abundances is important (within a factor of less than 100). This makes them very sensitive tracers. For Model 0, it is shown that $\mathrm{CN}, \mathrm{HNC}, \mathrm{HCN}, \mathrm{C}_{2}$, and $\mathrm{C}_{2} \mathrm{H}$ reach their highest relative abundances having in addition significant variations (factor $\gtrsim 10^{3}$ ) as compared to Model 9 (nitrogen-poor model). These last two species are seen particularly sensitive to the nitrogen gas content since they are likely to only survive in nitrogen-rich environment (for a $\mathrm{N} / \mathrm{H}$ ratio higher than $6.5 \times 10^{-5}$ ). The models having the highest $\mathrm{O} / \mathrm{H}$ and $\mathrm{S} / \mathrm{H}$ ratios do not reveal $\mathrm{H}_{2} \mathrm{CO}, \mathrm{H}_{2} \mathrm{CS}$, and $\mathrm{CH}_{2} \mathrm{CO}$ as particularly good tracers. Their corresponding relative abundances reach their lowest values for Models 10 and 11, while their maximum corresponds to Model 0 . The decrease in relative abundance seen for some of them, when the $\mathrm{O} / \mathrm{H}$ and $\mathrm{S} / \mathrm{H}$ elemental abundance ratios are increased, is so pronounced (e.g., up to a factor of 1000 for $\mathrm{H}_{2} \mathrm{CO}$ ) that the abundances fall under the limit of detectability. These molecules are thus likely to be undetectable in oxygen- and sulfur-rich primordial gas while potentially observable in nitrogen-rich environments. The trends we have identified are summarized in Table 5.

$$
\text { 4.3. } A_{v}
$$

As expected, and shown in Figure 3, the abundances of many molecular species are much smaller at an $A_{v}=3$ (Model 5 in 
Table 4

Trends of Molecular Fractional Abundances, Computed for Different Values of Metallicity ${ }^{\mathrm{a}}$

\begin{tabular}{ll}
\hline \hline Molecule & \multicolumn{1}{c}{ Response to Metallicity Changes } \\
\hline $\mathrm{CO}, \mathrm{H}_{2} \mathrm{O}$ & Linear tracers of metallicity \\
$\mathrm{CS}, \mathrm{SO}$ & For $z>1 / 100 z_{\odot}$, good linear tracers of metallicity \\
$\mathrm{CN}$ & For $z>1 / 100 z_{\odot}$, insensitive to metallicity changes \\
$\mathrm{OH}, \mathrm{H}_{3} \mathrm{O}^{+}$ & Most abundant at $z=1 / 100 z_{\odot}$; generally insensitive to $z$ \\
$\mathrm{HNC}, \mathrm{HCN}$ & For $z>1 / 10 z_{\odot}$, insensitive to metallicity changes \\
$\mathrm{HCO}^{+}$ & Always above the limit of detectability; insensitive to metallicity changes \\
$\mathrm{C}_{2}, \mathrm{C}_{2} \mathrm{H}$ & For $z>1 / 10 z_{\odot}$, inversely dependent on $z$ changes \\
$\mathrm{CO}_{2}, \mathrm{OCS}$ & Good linear tracers of metallicity except for $z<1 / 100 z_{\odot}($ undetectable) \\
$\mathrm{SO}_{2}, \mathrm{H}_{2} \mathrm{~S}$ & For $z>1 / 100 z_{\odot}$, good linear tracer of metallicity \\
$\mathrm{H}_{2} \mathrm{CS}, \mathrm{CH}$ & $\mathrm{CO}$ \\
$\mathrm{H}_{2} \mathrm{CO}$ & For $z>1 / 10 z_{\odot}$, inversely dependent on $z$ changes \\
\hline
\end{tabular}

Note. " When a single species is defined as "undetectable" or "not detectable," it means that its relative abundance is below the adopted limit of detectability of $1 \times 10^{-12}$.

Table 5

Trends of Molecular Fractional Abundances, Computed for Different Values of the Initial Elemental Abundance Ratios ${ }^{\mathrm{a}}$

\begin{tabular}{ll}
\hline \hline Molecule & \multicolumn{1}{c}{ Response to Initial Elemental Abundance Ratio Changes } \\
\hline $\mathrm{CO}, \mathrm{H}_{2} \mathrm{O}$ & Insensitive to changes of any initial elemental abundance ratios \\
$\mathrm{CS}$ & Sensitive to changes in both $\mathrm{S} / \mathrm{H}$ and $\mathrm{O} / \mathrm{H}$; variations within a factor of $\geqslant 50$ \\
$\mathrm{SO}$ & Very good tracer of $\mathrm{S} / \mathrm{H}$; variations up to a factor of $\approx 1000$; very sensitive to $\mathrm{O} / \mathrm{H}$ changes \\
$\mathrm{CN}$ & Highest abundance for $\mathrm{N}$-rich models; very good tracer of $\mathrm{N} / \mathrm{H}$ \\
$\mathrm{OH}$ & Insensitive to any changes in initial elemental abundance ratios \\
$\mathrm{HNC}, \mathrm{HCN}_{\mathrm{HCO}}^{+}, \mathrm{H}_{3} \mathrm{O}^{+}$ & Very good tracers of $\mathrm{N} / \mathrm{H}$ \\
$\mathrm{C}_{2}, \mathrm{C}_{2} \mathrm{H}$ & Poor tracer of initial elemental abundance ratios \\
$\mathrm{CO}_{2}$ & Surviving only if $\mathrm{N}$-rich environments \\
$\mathrm{OCS}_{\mathrm{SO}}$ & Insensitive to changes of any initial elemental abundance ratios \\
$\mathrm{H}_{2} \mathrm{~S}$ & Highest abundance for $\mathrm{S}$ - and O-rich models; variations within a factor of $\approx 100 ;$ good tracer \\
$\mathrm{H}_{2} \mathrm{CS}$ & Very good tracer of $\mathrm{S} / \mathrm{H}$ and O/H \\
$\mathrm{CH}_{2} \mathrm{CO}, \mathrm{H}_{2} \mathrm{CO}$ & Insensitive to any changes in initial elemental abundance ratios \\
\hline
\end{tabular}

Note. ${ }^{\text {a }}$ Comparison of the PDR chemistry for various values of initial abundances (see Table 3 and Models 9-11 in Table 2) while other parameters have the standard values (see Table 1).

Table 6

Trends of Molecular Fractional Abundances, Computed for Two Values of the Opacity $\left(A_{v}\right)^{\mathrm{a}}$

\begin{tabular}{ll}
\hline \hline Molecule & \multicolumn{1}{c}{ Response to Opacity Changes } \\
\hline $\mathrm{CO}$ & Insensitive to opacity changes \\
$\mathrm{H}_{2} \mathrm{O}, \mathrm{HNC}, \mathrm{HCN}, \mathrm{SO}$ & Very good tracers of $A_{v}$ \\
$\mathrm{CS}$ & At $A_{v}=3$, very good tracer of the opacity \\
$\mathrm{CN}, \mathrm{HCO}^{+}, \mathrm{H}_{3} \mathrm{O}^{+}$ & Not very sensitive to opacity changes \\
$\mathrm{OH}$ & Insensitive to opacity changes; poor tracer of $A_{v}$ \\
$\mathrm{C}_{2}, \mathrm{C}_{2} \mathrm{H}$ & Insensitive to opacity changes \\
$\mathrm{CO}_{2}$ & At $A_{v}=3$, abundance decreases by a factor of $10^{4} ;$ very good tracer of $A_{v}$ \\
$\mathrm{OCS}_{2} \mathrm{H}_{2} \mathrm{CS}$ & At $A_{v}=3$, reduced by a factor of $10^{4} ;$ become undetectable \\
$\mathrm{SO}_{2}$ & At $A_{v}=3$, reduced by a factor of $10^{6} ;$ become undetectable \\
$\mathrm{H}_{2} \mathrm{~S}, \mathrm{CH} \mathrm{CH}_{2} \mathrm{CO}$ & At $A_{v}=3$, decreased by a factor of $\geqslant 10^{2} ;$ become undetectable \\
$\mathrm{H}_{2} \mathrm{CO}$ & When $A_{v}=3$, abundance reduction by a factor of $10 ;$ become undetectable
\end{tabular}

Note. ${ }^{\text {a }}$ Comparison of the PDR chemistry for $A_{v}=8$ (Model 0) and $A_{v}=3$ (Model 5), while other parameters have the standard values (see Table 1).

Table 2) than at $A_{v}=8$ (Model 0 in Table 2). For example, $\mathrm{H}_{2} \mathrm{O}$, $\mathrm{HNC}$, and SO molecules show fractional abundances smaller by more than 2 orders of magnitude at $A_{v}=3$ than at $A_{v}=8$. For $\mathrm{CS}$ and $\mathrm{SO}_{2}$, the reduction factors are even larger (see Figure 3 and Table 6). These species are very sensitive to $A_{v}$ and good tracers of opacity. At $A_{v}=3$, however, some species become undetectable since their corresponding relative abundance drops below the probable limit of detectability.

The strong response to $A_{v}$ is due to the ability of FUV photons to penetrate the translucent cloud conditions $\left(A_{v} \approx 3\right)$ but not much deeper. $\mathrm{CO}$ is self-shielding and generally insensitive for $A_{v} \gtrsim 1$. Some other molecules $\left(\mathrm{C}_{2}, \mathrm{C}_{2} \mathrm{H}\right)$ depend on dissociative ionization of $\mathrm{CO}$, and so are also fairly insensitive to $A_{v}$.

\subsection{Cosmic Ray Ionization Rate}

Using Table 7 and Figure 4, we identify five groups of species showing various degrees of sensitivity to the change in the cosmic ray ionization rate (from Model 0 with $\zeta=5 \times 10^{-17} \mathrm{~s}^{-1}$ to Model 6 with $\zeta=5 \times 10^{-15} \mathrm{~s}^{-1}$, see Table 2). The first group, 


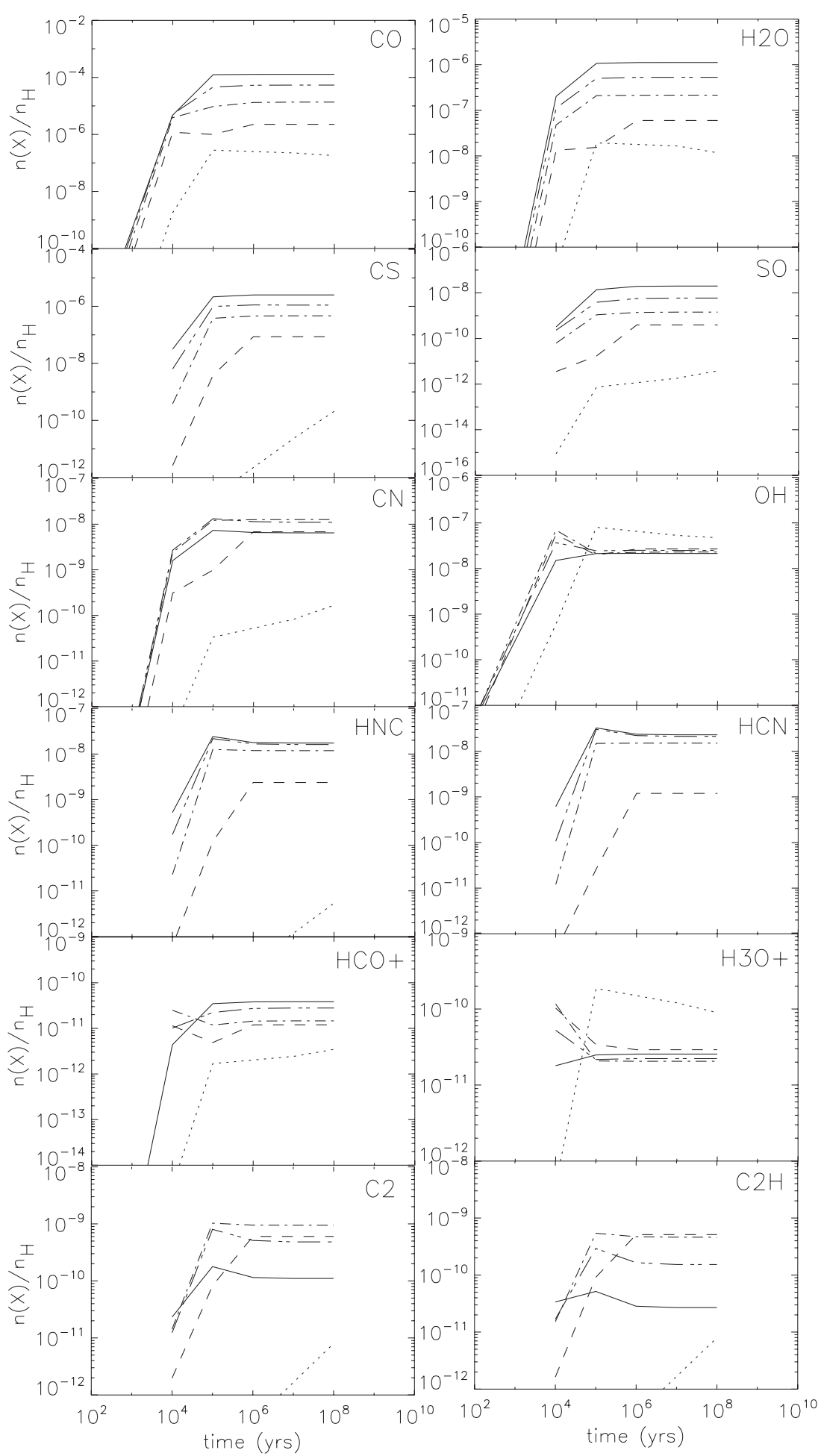

Figure 1. Influence of metallicity variations on some molecular abundances. We plot in a logarithmic scale the relative abundances $n(\mathrm{X}) / n_{\mathrm{H}}$ of various species, $\mathrm{X}$, with respect to the time (in yr). Results from the first five models in Table 2 (Models $0-4$ ) are plotted in this figure: Model 0 (solar metallicity) is represented by black lines, Model $1\left(1 / 2 z_{\odot}\right)$ by dash-two-dots lines, while Models $2\left(1 / 4 z_{\odot}\right), 3\left(1 / 10 z_{\odot}\right)$, and $4\left(1 / 100 z_{\odot}\right)$ are symbolized by dash-one-dot lines, dashed lines, and dotted lines, respectively, showing the effect of decreasing the metallicity.

including OCS, $\mathrm{SO}_{2}$, or $\mathrm{H}_{2} \mathrm{CS}$, contains the most sensitive species. Indeed these molecules have their respective chemical abundances reduced by a factor of $\sim 10^{4}$ when $\zeta$ increases by 2 orders of magnitude. This approximately quadratic response to the enhancement in $\zeta$ make them very good tracers of cosmic rays, especially in very active star-forming regions or where an AGN contribution occurs. However, this very high sensitivity leads certain species to become undetectable at high $\zeta$. This is the case for OCS and $\mathrm{SO}_{2}$ whose relative abundances thus fall below our (arbitrary) limit of detectability of $1 \times 10^{-12}$ for models with $\zeta=5 \times 10^{-15} \mathrm{~s}^{-1}$.

A second category contains species such as $\mathrm{SO}, \mathrm{H}_{2} \mathrm{~S}, \mathrm{CH}_{2} \mathrm{CO}$, $\mathrm{CS}, \mathrm{HNC}$, or HCN whose abundances respond approximately linearly to changes in $\zeta$. These species are good tracers of 

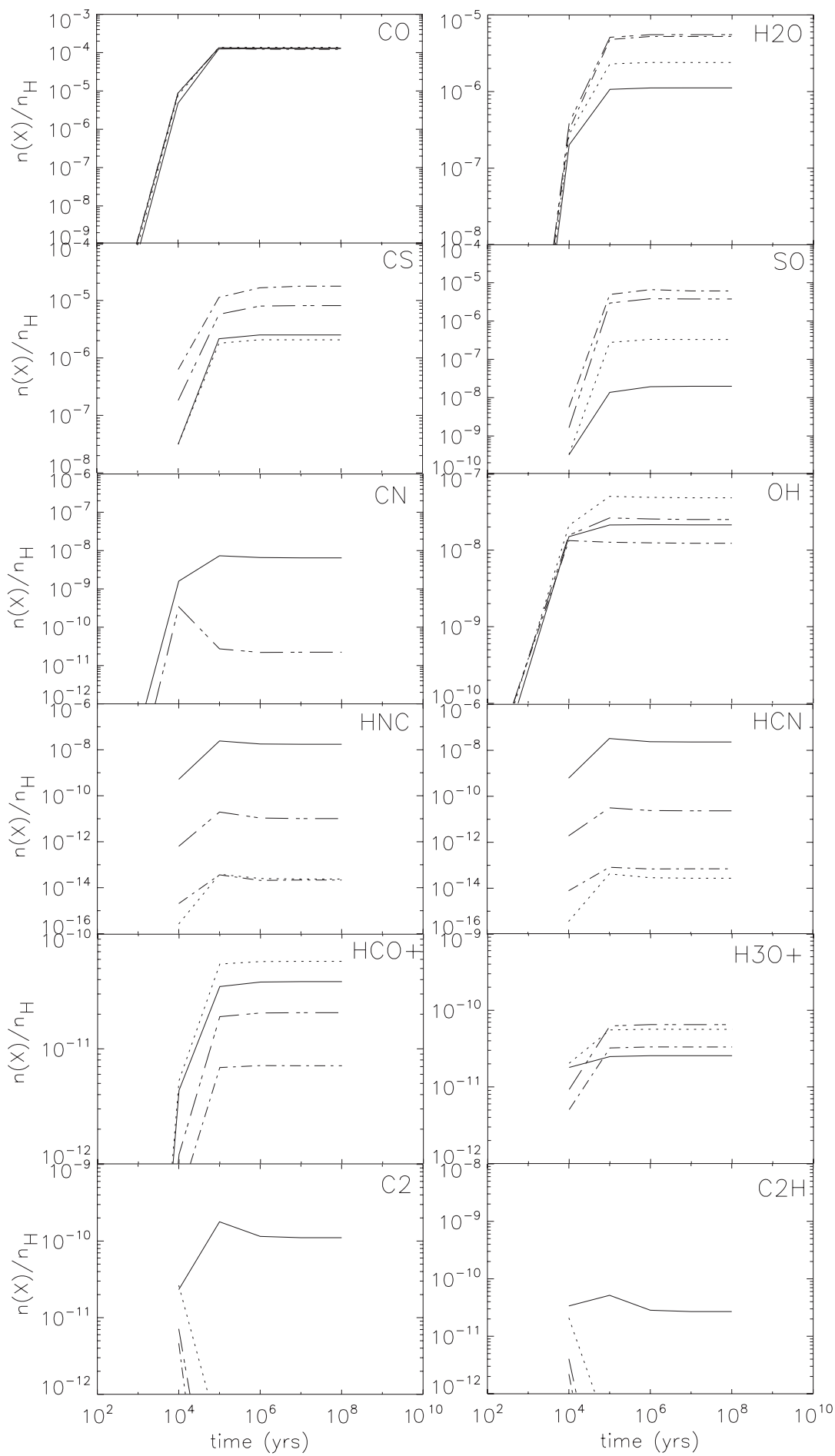

Figure 2. Influence of initial elemental abundance ratio variations on some molecular abundances. We plot in a logarithmic scale the relative abundances $n(\mathrm{X}) / n_{\mathrm{H}}$ of various species, X, with respect to the time (in yr). Only the solar (Model 0) and the last three models from Table 2 (Models 9-11) are plotted. Model 0 is represented by black lines, Model 9 by dotted lines, while Models 10 and 11 are symbolized by dash-one-dot lines, dash-two-dot lines, respectively. The values of the initial elemental abundance ratios for each model are given in Table 3.

cosmic rays as long as their fractional abundances are above the detectability limit.

A third category of species includes molecules such as $\mathrm{CO}$ or $\mathrm{H}_{2} \mathrm{O}$ which show modest reduction of their chemical abundances to increases in $\zeta$.

Species such as $\mathrm{CN}, \mathrm{OH}$, or $\mathrm{HCO}^{+}$are fairly insensitive to changes in $\zeta$.

Finally, we identify species whose fractional abundance increases slightly (by a factor of $\sim 10$; such as $\mathrm{H}_{3} \mathrm{O}^{+}$) or more heavily (by a factor $\sim 100$; such as $\mathrm{C}_{2}$ and $\mathrm{C}_{2} \mathrm{H}$ ) in response to an increase of $\zeta$. Ratios of abundances of molecules from the first and fifth groups would be highly sensitive tracers of $\zeta$.

The higher value of $\zeta$ used here generates loss rates for most species that exceed other loss rates, and hence most abundances decrease for higher $\zeta$. However, the larger $\zeta$ also tends to increase the rate of forming of ions such as $\mathrm{C}^{+}$, so that transient species such as $\mathrm{C}_{2}$ and $\mathrm{C}_{2} \mathrm{H}$ increase. 


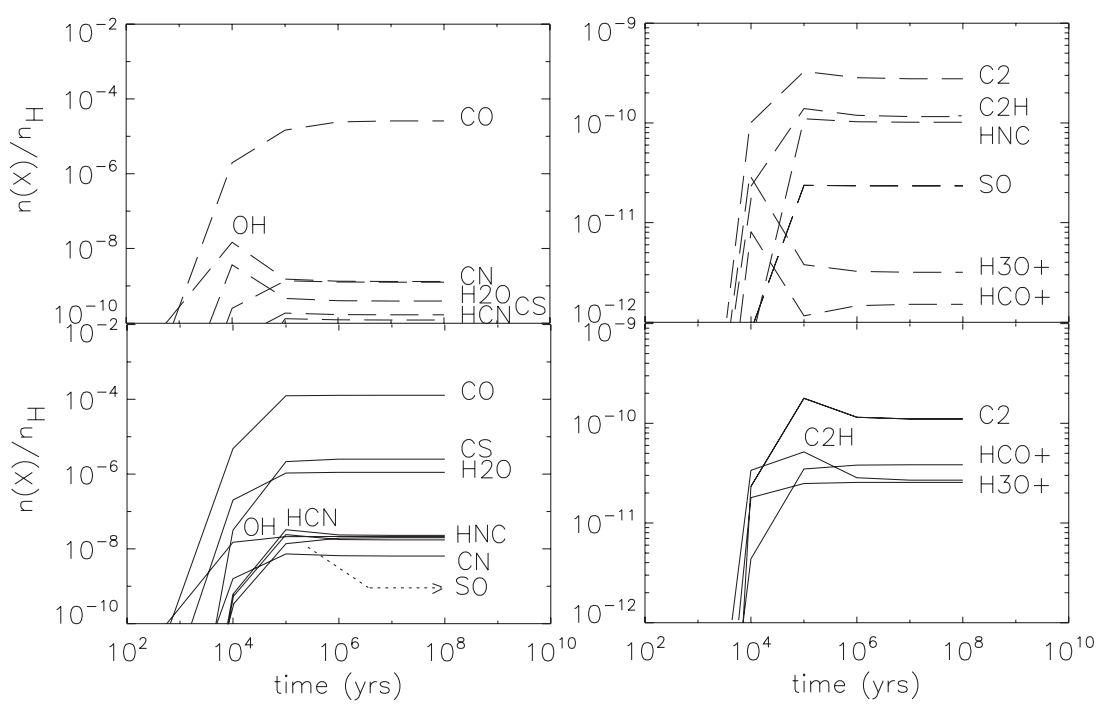

Figure 3. Influence of $A_{v}$ variations on some molecular abundances. We plot in a logarithmic scale the relative abundances $n(\mathrm{X}) / n_{\mathrm{H}}$ of various species, $\mathrm{X}$, with respect to the time (in yr). The upper two panels (dashed lines) are dedicated to Model 5 ( $A_{v}=3$ in Table 2), while the lower two plots (black lines) present the results of the solar model (Model 0 in Table 2) for $A_{v}=8$.

Table 7

Trends of Molecular Fractional Abundances, Computed for Different Values of the Cosmic Ray Ionization Rate $(\zeta)^{\mathrm{a}}$

\begin{tabular}{ll}
\hline \hline Molecule & \multicolumn{1}{c}{ Response to Cosmic Ray Ionization Rate Changes } \\
\hline $\mathrm{CO}, \mathrm{H}_{2} \mathrm{O}$ & Reduced by a factor of 10 with $\zeta$; possible tracers of $\zeta$ \\
$\mathrm{CS}, \mathrm{SO}, \mathrm{HNC}, \mathrm{HCN}$ & Heavy (linear) abundance reduction by a factor of 100 with $\zeta$; good tracers of $\zeta$ \\
$\mathrm{CN}, \mathrm{OH}, \mathrm{HCO}^{+}$ & Insensitive to $\zeta$ \\
$\mathrm{H}_{3} \mathrm{O}^{+}$ & Slight increase by a factor of 10 with $\zeta$ \\
$\mathrm{C}_{2}, \mathrm{C}_{2} \mathrm{H}$ & Heavy (linear) abundance increase by a factor of 100 with $\zeta$ \\
$\mathrm{CO}_{2}, \mathrm{H}_{2} \mathrm{CS}$ & Very heavy reduction by a factor of $\sim 10^{4}$; very good tracer of $\zeta$ \\
$\mathrm{OCS}_{2} \mathrm{SO}_{2}$ & Very heavily reduced by a factor of $\sim 10^{4}$ with $\zeta$; become undetectable \\
$\mathrm{H}_{2} \mathrm{~S}, \mathrm{CH}_{2} \mathrm{CO}$ & Heavy (linear) abundance reduction by a factor of 100 with $\zeta$; become undetectable \\
$\mathrm{H}_{2} \mathrm{CO}$ & Insensitive to $\zeta$ \\
\hline
\end{tabular}

Note. ${ }^{\text {a}}$ Comparison of the PDR chemistry for $\zeta=1 \zeta_{\odot}=5 \times 10^{-17} \mathrm{~s}^{-1}$ (Model 0 ) and $\zeta=100 \times \zeta_{\odot}=5 \times 10^{-15} \mathrm{~s}^{-1}$ (Model 6), while other parameters have the standard values (see Table 1).

Table 8

Trends of Molecular Fractional Abundances, Computed for Different Values of the FUV Radiation Field ${ }^{\text {a }}$

\begin{tabular}{ll}
\hline \hline Molecule & \multicolumn{1}{c}{ Response to FUV Radiation Field Changes } \\
$\mathrm{CO}$ & Insensitive to FUV Changes \\
$\mathrm{H}_{2} \mathrm{O}$ & Reduced by a factor of 100 (linearly to the FUV change); good tracer \\
$\mathrm{CS}$ & Heavy abundance reduction by a factor of 1000 with FUV; very sensitive to FUV changes \\
$\mathrm{SO}$ & Moderately reduced by a factor of 10 with FUV; possible tracer of FUV \\
$\mathrm{CN}, \mathrm{OH}, \mathrm{HNC}$, & Insensitive to the changes in FUV \\
$\mathrm{HCN}, \mathrm{HCO}^{+}, \mathrm{H}_{3} \mathrm{O}^{+}$ & Insensitive to the changes in FUV \\
$\mathrm{C}_{2}, \mathrm{C}_{2} \mathrm{H}$ & Slight abundance increase by a factor of $\leqslant 10$ with FUV changes \\
$\mathrm{CO}_{2}, \mathrm{H}_{2} \mathrm{~S}, \mathrm{H}_{2} \mathrm{CS}$ & Abundance decreases (linearly) by a factor of 100 with the FUV changes; good tracers \\
$\mathrm{OCS}_{2} \mathrm{SO}_{2}$ & Heavy reduction by a factor of $1000 ;$ very sensitive to FUV changes; very good tracer \\
$\mathrm{CH}_{2} \mathrm{CO}, \mathrm{H}_{2} \mathrm{CO}$ & Insensitive to FUV changes \\
\hline
\end{tabular}

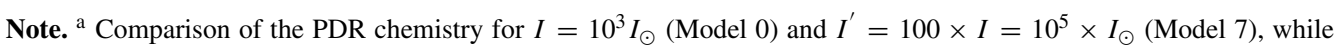
other parameters have the standard values (see Table 1).

\subsection{FUV Radiation Field}

As expected, increasing the FUV radiation field by 2 orders of magnitude leads to a general decrease of molecular abundances (see Figure 5 comparing Models 0 and 7 listed in Table 2; see also Table 8). Sulfur-bearing species also appear very sensitive to the changes in the radiation field which makes them very good tracers of FUV flux. Molecules such as $\mathrm{H}_{2} \mathrm{~S}$ or $\mathrm{H}_{2} \mathrm{CS}$ show relative abundances linearly reduced with respect to the FUV increase, while the CS, OCS, and $\mathrm{SO}_{2}$ molecules are more heavily reduced by a factor of $\sim 1000$. However, $\mathrm{SO}$ is less sensitive. Molecules $\mathrm{CO}, \mathrm{CN}, \mathrm{OH}, \mathrm{HNC}, \mathrm{HCN}, \mathrm{HCO}^{+}$, and $\mathrm{H}_{3} \mathrm{O}^{+}$seem to be rather insensitive to the changes in the FUV radiation field. 

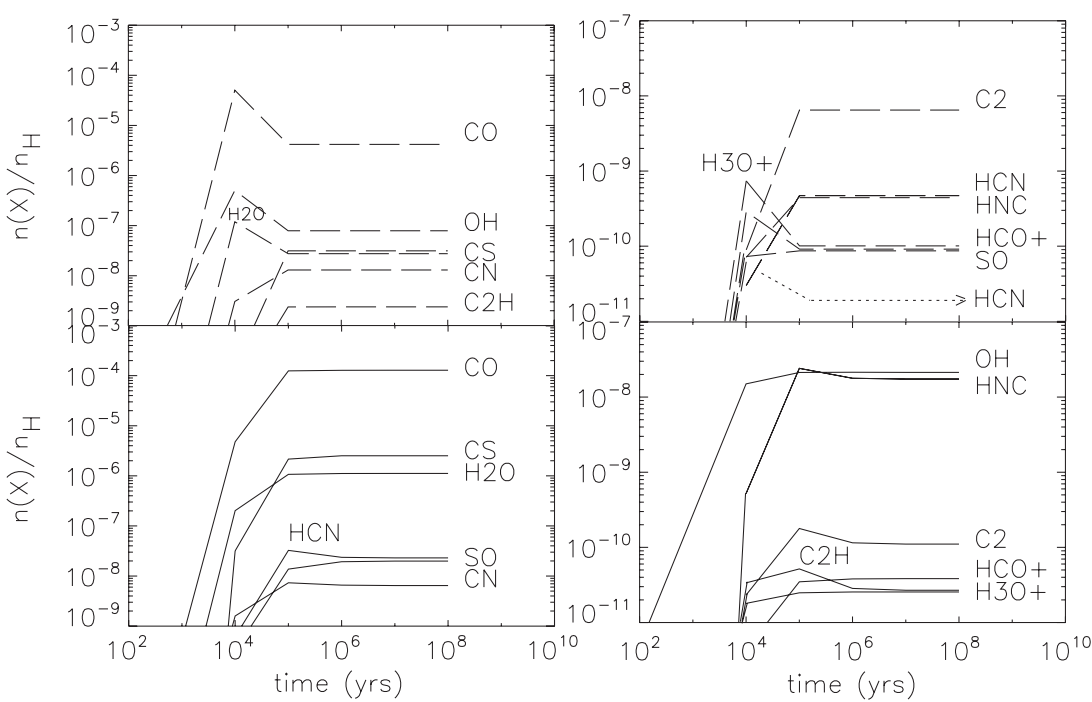

Figure 4. Influence of cosmic ray ionization rate variations on some molecular abundances. We plot in a logarithmic scale the relative abundances $n(\mathrm{X}) / n_{\mathrm{H}}$ of various species, X, with respect to the time (in yr). The upper two panels (dashed lines) are dedicated to Model 6 ( $\zeta$ enhanced 100 times), while the lower two plots (black lines) show results for Model 0.

Table 9

Trends of Molecular Fractional Abundances, Computed for Different Values of the Density ${ }^{\mathrm{a}}$

\begin{tabular}{|c|c|}
\hline Molecule & Response to Density Changes \\
\hline $\mathrm{CO}, \mathrm{H}_{2} \mathrm{O}, \mathrm{CS}, \mathrm{HNC}, \mathrm{HCN}$ & Abundance variations within a factor of $\leqslant 4$; poor tracers \\
\hline SO & Abundance increase by a factor of $\sim 10$ with density; possible tracer of density \\
\hline $\mathrm{CN}, \mathrm{C}_{2}$ & Decrease by a factor of 100 (linearly inverse with the change of density); very good tracer \\
\hline $\mathrm{OH}$ & Decrease by a factor of $\sim 10$; possible tracer of density \\
\hline $\mathrm{HCO}^{+}, \mathrm{H}_{3} \mathrm{O}^{+}$ & Abundance variations within a factor of $\leqslant 4$; insensitive to density changes \\
\hline $\mathrm{C}_{2} \mathrm{H}$ & Decrease by a factor of $\geqslant 100$; undetectable at high gas density \\
\hline $\mathrm{CO}_{2}, \mathrm{OCS}, \mathrm{H}_{2} \mathrm{~S}$ & Increase by a factor of $\geqslant 100 ;$ very good (linear) tracers of $n\left(\mathrm{H}_{2}\right)$ \\
\hline $\mathrm{SO}_{2}$ & Increase by a factor of $\geqslant 1000$; very good (quadratic) tracer of $n\left(\mathrm{H}_{2}\right)$ \\
\hline $\mathrm{H}_{2} \mathrm{CS}$ & Abundance variations within a factor of $\sim 2$; insensitive to density changes \\
\hline $\mathrm{CH}_{2} \mathrm{CO}$ & Decrease by a factor of $\geqslant 10^{5}$; undetectable at high density \\
\hline $\mathrm{H}_{2} \mathrm{CO}$ & (Linear) decrease by a factor of $\geqslant 100$; undetectable at $n\left(\mathrm{H}_{2}\right)=10^{6} \mathrm{~cm}^{-3}$ \\
\hline
\end{tabular}

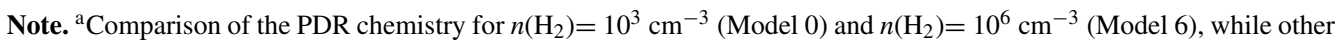
parameters have the standard values (see Table 1).

The small increase of the relative abundances of molecules such as $\mathrm{C}_{2}$ and $\mathrm{C}_{2} \mathrm{H}$ is due to the injection of free carbon from the photodestruction of carbon-bearing species. This injection stimulates hydrocarbon growth.

\subsection{Density}

We can roughly separate species into four main categories, depending on their behavior when the density changes. Using Figure 6 and Table 9, we identify a first category including species rather insensitive to gas density changes. This includes molecules such as $\mathrm{CO}, \mathrm{H}_{2} \mathrm{O}, \mathrm{H}_{2} \mathrm{CS}, \mathrm{CS}, \mathrm{HCO}^{+}, \mathrm{H}_{3} \mathrm{O}^{+}, \mathrm{HCN}$, or $\mathrm{HNC}$ whose abundance variations are below a factor of $\sim 5$. This category is followed by the molecules $\mathrm{SO}$ and $\mathrm{OH}$ which show moderate change in their chemical abundances by a factor of $\approx 10$ (especially after $10^{6} \mathrm{yr}$, see Figure 6). A third category includes species that respond to an increase in gas number density from $10^{4}$ to $10^{6} \mathrm{~cm}^{-3}$ with heavy reduction ( $\mathrm{CN}$ and $\mathrm{C}_{2}$, factors of 100 ), and very heavy reduction of their fractional abundances $\left(\mathrm{C}_{2} \mathrm{H}, \mathrm{CH}_{2} \mathrm{CO}\right.$, or $\mathrm{H}_{2} \mathrm{CO}$, factors of greater than 100). These very heavy reductions usually lead to species becoming nondetectable (see Table 9). For this reason, $\mathrm{C}_{2} \mathrm{H}, \mathrm{CH}_{2} \mathrm{CO}$, and $\mathrm{H}_{2} \mathrm{CO}$ cannot be considered as good tracers of density. On the contrary, $\mathrm{CN}$ and $\mathrm{C}_{2}$ molecules could be seen as very good tracers of gas density since they are linearly linked with density changes. A last group of species able to be recognized as very good tracers of density, contains the molecules $\mathrm{CO}_{2}, \mathrm{OCS}, \mathrm{H}_{2} \mathrm{~S}$, and $\mathrm{SO}_{2}$. The relative abundances of these species indeed vary linearly (a factor of 100) with the change in gas density, except for the $\mathrm{SO}_{2}$ which varies quadratically with the gas density, promoting this molecule to the best (detectable) tracer of $n\left(\mathrm{H}_{2}\right)$.

However, these results take no account of the fact that freezeout of gas-phase species has been omitted from these models. For gas of number density $n_{\mathrm{H}}$ and metallicity $z$, the freezeout timescale is $\approx 10^{6} \mathrm{yr}\left(10^{4} / n_{\mathrm{H}}\right)\left(z_{\odot} / z\right)$. Thus, at $n_{\mathrm{H}}=10^{4} \mathrm{~cm}^{-3}$ and $z=z_{\odot}$ the timescale is around one million years, a plausible lifetime for gas in a PDR. However, at $n_{\mathrm{H}}=10^{6} \mathrm{~cm}^{-3}$, the timescale becomes unfeasibly short unless the metallicity is very low. Thus, unless desorption processes are operating efficiently (Roberts et al. 2007) significant freezeout will be occurring in gas at very high densities.

\section{PREDICTED PDR TRACERS FOR SELECTED GALAXY TYPES}

In this section, we have selected several fairly well-known galaxies as illustrating examples of the $\mathrm{SB}, \mathrm{SB}+\mathrm{AGN}$, and 

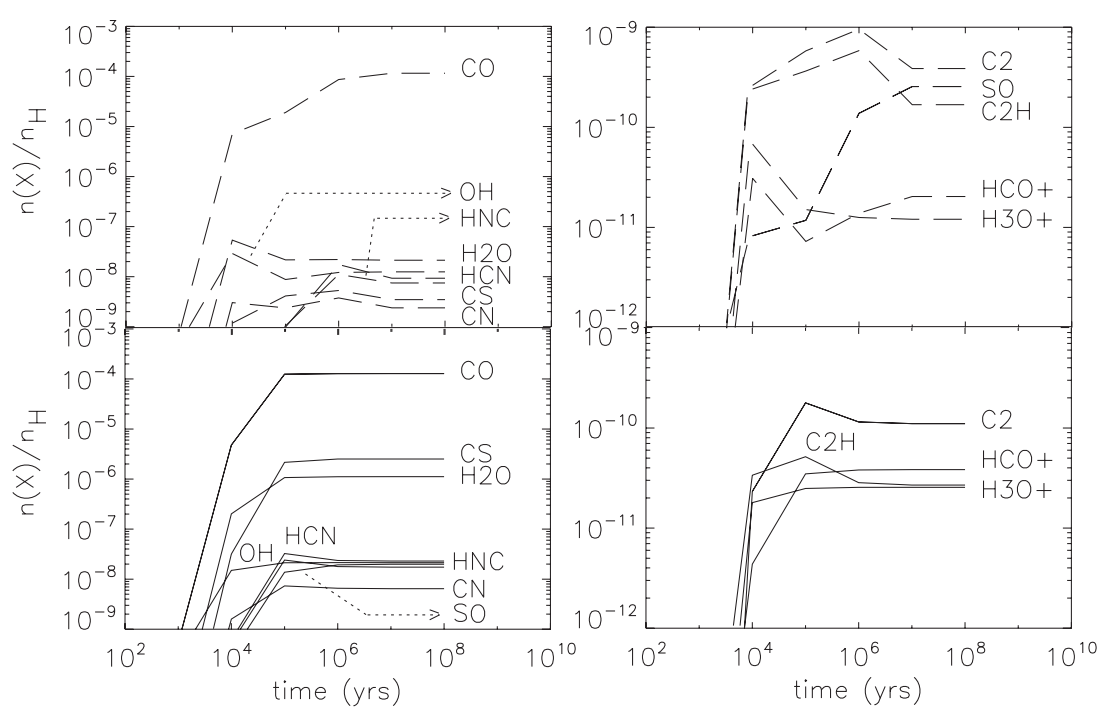

Figure 5. Influence of FUV radiation field variations on some molecular abundances. We plot in a logarithmic scale the relative abundances $n(\mathrm{X}) / n_{\mathrm{H}}$ of various species, $\mathrm{X}$, with respect to the time (in yr). The upper two panels (dashed lines) are dedicated to Model 7 (FUV radiation field enhanced 100 times), while the lower two plots (black lines) show results for Model 0.
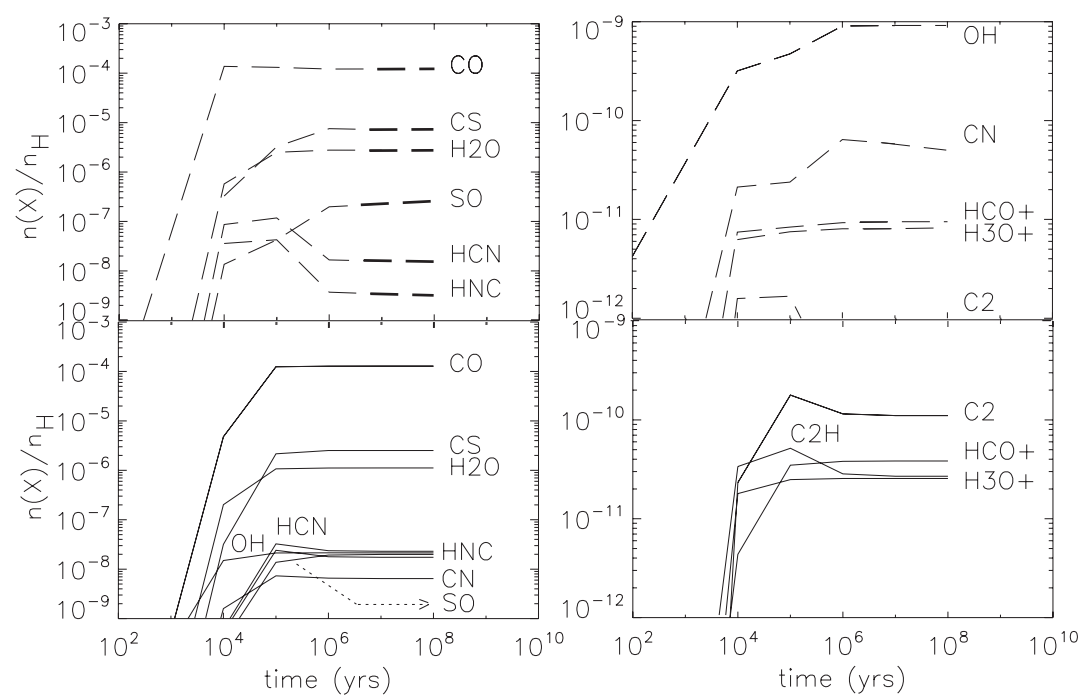

Figure 6. Influence of density variations on some molecular abundances. We plot in a logarithmic scale the relative abundances $n(\mathrm{X}) / n_{\mathrm{H}}$ of various species, $\mathrm{X}$, with respect to the time (in yr). The upper two panels (dashed lines) are dedicated to Model 8 (number density of $10^{6} \mathrm{~cm}^{-3}$ ), while the lower two plots (black lines) show results for Model 0.

high - $z$ galaxies. These galaxies are compared with our selection of models in an attempt to identify, for each case, the best molecular tracers of dense PDR gas. This study is of particular interest for the preparations of the future observational programs of Herschel and ALMA.

An interesting type of galaxy concerns objects such as M 83, NGC 253, i.e., typical SBs. It is commonly accepted that one may reproduce such environments by using an FUV radiation field enhanced as compared to normal spiral galaxies (Israel \& Baas 2001; Bayet et al. 2006, 2008b). M 83 and NGC 253 show solar metallicity (see Origlia et al. 2004; Smith et al. 2006 and Zaritsky et al. 1994, respectively), leading to a choice of standard elemental initial abundance ratios and density. Model 7 appears consequently a good model likely to represent this morphological type of galaxy.

Another morphological type of galaxy that we have investigated concerns galaxies hosting an additional source such as active nucleus ( $\mathrm{SB}+\mathrm{AGN})$. Objects such as the galaxies Arp 220 (Soifer et al. 1984; Joy et al. 1986) or NGC 3079 (Ford et al. 1986; Sosa-Brito et al. 2001) are well-known examples of this category. Indeed, whether Arp 220 has an AGN or not is still a very highly debated topic (see, for instance, Downes \& Eckart 2007; Sakamoto et al. 2008; Aalto et al. 2008). Although NGC 3079 certainly contains an AGN, it seems that also for this source the more extended SB may dominate the molecular gas properties (see, for instance, Kohno et al. 2007). Following the parameter choices from Bayet et al. (2008b), we have adopted here Model 6 to represent these environments. Indeed, in this model, the cosmic ray ionization rate is enhanced 100 times $\left(\zeta=5 \times 10^{-15} \mathrm{~s}^{-1}\right)$ as compared to the standard values, while the FUV radiation has been maintained at $10^{3} I_{\odot}$. The selected metallicity is solar. The density, the initial elemental abundance ratios and the $A_{v}$ values are standard. The model adopted here is thus an attempt to simulate qualitatively XDR-like environments. To reproduce "pure" AGN sources, full XDR models (Meijerink et al. 2005; Meijerink \& Spaans 2005; Spaans \& Meijerink 2005) are however required. 
Table 10

Detectability of 19 Species Likely to Trace the Dense PDR Gas Component in Three Categories of Galaxies (see Section 5) ${ }^{\mathrm{a}}$

\begin{tabular}{lccc}
\hline \hline Galaxy Type & Model 7 & Model 6 & Model 12 \\
\hline $\mathrm{CO}$ & $\mathrm{SB}$ & $\mathrm{SB}+$ AGN & High Redshift \\
$\mathrm{H}_{2} \mathrm{O}$ & + & + & + \\
$\mathrm{CS}$ & + & + & + \\
$\mathrm{SO}$ & + & + & + \\
$\mathrm{CN}$ & + & + & + \\
$\mathrm{OH}$ & + & + & + \\
$\mathrm{HNC}$ & + & + & + \\
$\mathrm{HCN}$ & + & + & + \\
$\mathrm{HCO}$ & + & + & + \\
$\mathrm{H}_{3} \mathrm{O}^{+}$ & + & + & + \\
$\mathrm{C}_{2}$ & + & + & + \\
$\mathrm{C}_{2} \mathrm{H}$ & + & + & + \\
$\mathrm{CO}_{2}$ & + & + & + \\
$\mathrm{OCS}$ & + & - & + \\
$\mathrm{SO}_{2}$ & + & - & - \\
$\mathrm{H}_{2} \mathrm{~S}$ & - & - & - \\
$\mathrm{H}_{2} \mathrm{CS}$ & - & + & - \\
$\mathrm{H}_{2} \mathrm{CO}$ & + & + & - \\
$\mathrm{CH}_{2} \mathrm{CO}$ & + & - & - \\
\hline
\end{tabular}

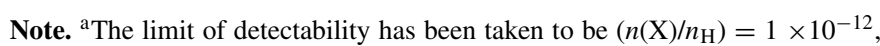
as is typical for molecules in the Milky Way. Under this limit the species are not detectable (symbol -). Otherwise, they are marked with the symbol +. We have separated the species plotted in Figures 1 and 2 from those only listed in Tables 4 and 5 .

Finally, we want to investigate galaxies at high redshift. Typical examples to be considered are the Cloverleaf QSO and APM 08279 with their respective redshifts of $z_{\text {red. }} \approx 2.6$ and $z_{\text {red. }} \approx 3.9$. For these sources, the physical properties are unknown or highly uncertain, even if several molecules have already been detected (García-Burillo et al. 2006; Guélin et al. 2007; Krips et al. 2007; Weiß et al. 2007; Riechers et al. 2009). The presence of both dense and less dense gas makes these sources particularly good illustrating examples for our study. Some of these measurements (Gao \& Solomon 2004; Wu et al. 2005; Wagg et al. 2005) suggest that star formation may be active, even if these galaxies are affected by an AGN. GarcíaBurillo et al. (2006) have suggested that the unusual chemistry observed may be due to a combination of star formation and AGN (PDR/XDR) chemistries. In an attempt to describe such sources, we have run an additional model presented in Table 2 (Model 12). This model shows a high value of the cosmic ray ionization rate, a high value of the radiation field intensity, and a relatively low metallicity (all with respect to the Milky Way).

We summarize in Table 10 the species predicted to be the best molecular tracers of dense PDR gas in each case (above the limit of detectability arbitrarily taken to be equivalent to a fractional abundance of $1 \times 10^{-12}$ ). Here, our goal was to give the reader some observational clues derived from this modeling work. Our choice of galaxies are simply examples; none of the models we are presenting in Table 10 are meant as detailed studies of own individual galaxy. Together with the overall trends listed in Section 4, Table 10 should enable a more detailed description of the physical properties of the galaxy to be made.

Whichever model $\left(6,7\right.$, or 12) is used, $\mathrm{CO}$ and $\mathrm{H}_{2} \mathrm{O}$ molecules are predicted to have high fractional abundances (from $\approx$ $3 \times 10^{-8}$ up to $10^{-4}$ ). More surprisingly, for Models 6 and $7, \mathrm{CS}$, $\mathrm{CN}$, and $\mathrm{OH}$ also show relatively high fractional abundances, ranging between $10^{-9}$ and $10^{-8}$. According to the estimates of Lintott et al. (2005) these abundances are sufficiently large that unresolved active galaxies should be detectable in these species. Observational tests of the predictions listed in Table 10 have been already performed by Bayet et al. (2008a). They have revealed the importance of CS lines in SB galaxies. Further observational tests of predictions made in Table 10 will be detailed in forthcoming papers. For Model 12, CN and CS have chemical abundances which drop severely by at least 2 orders of magnitude, while the $\mathrm{OH}$ relative abundance increases by a factor of 10 relative to Model 0 . This feature again reinforces the interest of detecting these species, especially at high redshift.

Finally, it is interesting to note that molecules such as $\mathrm{HCN}, \mathrm{HNC}$, and $\mathrm{HCO}^{+}$do not show especially high relative abundances in the models investigated in this section. Indeed, the $\mathrm{HCN}$ and $\mathrm{HNC}$ chemical abundances for Models 6, 7, and 12 fall to $\approx 10^{-11}$. Moreover, the $\mathrm{HCO}^{+}$abundance varies from $2.0 \times 10^{-11}$ (Model 7) to $4.0 \times 10^{-10}$ (Model 12). However, we note that using a full XDR model (Meijerink \& Spaans 2005; Spaans \& Meijerink 2005; Meijerink et al. 2007) might change the results of Model 6. Indeed, in a full XDR model the cosmic rays considered are much more energetic than the ones normally used in PDR models.

\section{DISCUSSION AND CONCLUSIONS}

The main results of this study are the trends in the chemistry in response to changes in the physical conditions (model parameters) summarized in Tables 4-9. In addition to the evolution of the chemistry, Table 10 may be important in the context of the preparation of future observational programs (Herschel, ALMA). Our results focus mainly on the chemistry taking place at $A_{v} \approx 8$, i.e., in the densest part of the PDR. Analysis and discussion on the chemistry taking place in the outer region of the cloud $\left(A_{v} \ll 3\right)$ where the gas temperature and the FUV radiation field are higher is beyond the scope of this paper. While previous studies (Israel et al. 1995; Gerin \& Phillips 2000; Israel \& Baas 2001, 2002, 2003; Bayet et al. $2004,2006)$ focused their attention on determining the physical conditions of a few extragalactic star-forming or active regions using various PDR codes and the emissions of only a couple of molecules (mainly neutral carbon and carbon monoxide), we provide here a wide and complete analysis of the chemistry, and report in details on 19 species in a large range of parameter space.

Our model predictions must be compared with observations (e.g., Radford et al. 1991; Solomon et al. 1992, 2004; Martín et al. 2005, 2006; Evans et al. 2006; Graciá-Carpio et al. 2006; Aalto et al. 2007; Imanishi et al. 2007; Knudsen et al. 2007; Papadopoulos 2007) to be validated. Recent extragalactic detections of $\mathrm{HCN}$ and $\mathrm{HCO}^{+}$in 12 nearby galaxies summarized in Krips et al. (2008) provide useful estimations of molecular abundances. When the source hosts an AGN contribution, the relative $\mathrm{HCN}$ abundance they predicted from an large-scale velocity gradient (LVG) analysis of their data, is in agreement with our predictions (Model 6), to within a factor of 10. For SB-dominated galaxies, we also obtain a good agreement. As another example, $\mathrm{H}_{3} \mathrm{O}^{+}$has been recently detected in Arp 220 (van der Tak et al. 2008). The $\mathrm{H}_{3} \mathrm{O}^{+}$abundance in this $\mathrm{SB}+\mathrm{AGN}$ source is estimated to be $2-10 \times 10^{-9}$. Model 6 predicts an abundance of $\mathrm{H}_{3} \mathrm{O}^{+}$lower by a factor of 10 . However, van der Tak et al. (2008) mentioned that their abundance estimation is somewhat hampered by source size uncertainties, which we 
think may explain the discrepancy between our predictions and their observations. We conclude that our models appear to be in harmony with the limited observational data.

It is also particularly useful to compare the molecular tracers predicted by dense star-forming core models (Bayet et al. 2008b) with those presented in this article (derived from the UCL_PDR code). Indeed, it is essential for better understanding and diagnosing the nuclear energy source and activity in galaxies to be able to disentangle the PDR emission from that produced by dense star-forming cores. This should lead to a better determination of the excitation conditions in such regions. These studies are of importance because they concern not only the local universe (Kohno et al. 2001; Graciá-Carpio et al. 2006; Baan et al. 2008; Krips et al. 2008) but also could help us to better understand the galaxy formation in the universe at higher redshifts (Ivison et al. 2005a, 2005b, 2008; Younger et al. 2008a, 2008b). Comparing model predictions we thus have obtained that for the SB galaxy case, $\mathrm{SO}_{2}$ and $\mathrm{H}_{2} \mathrm{~S}$ are predicted to be likely inappropriate tracers of PDR regions, while they are predicted as good tracers of dense star-forming cores. By contrast, $\mathrm{HCO}^{+}$is predicted to be undetectable in dense starforming cores while enhanced in PDR-dominated galaxies. For high redshift sources, $\mathrm{H}_{2} \mathrm{CS}$ and $\mathrm{H}_{2} \mathrm{CO}$ are both predicted to be inadequate molecular tracers of PDRs, while both are good signatures of dense star-forming cores.

By comparing the model predictions for SB galaxies (Bayet et al. 2008b) and for the present study of PDR chemistry, we note that while the molecular component of AGN-excited galaxies is typical of PDRs, galaxies dominated by clusters of massive stars (SB) contain molecular tracers of both PDR and dense star-forming gas. Thus, in principle, it should be possible to distinguish between $\mathrm{SB}+\mathrm{AGN}$ and $\mathrm{SB}$ excitations.

In summary, we have explored PDR chemistry for a range of parameters representing galaxies with intense FUV or cosmic ray sources. Our main results demonstrate the sensitivity of the chemistry to the local physical conditions, and show that molecular observations can be used to determine those conditions. We have adopted very crude estimations of the relevant physical conditions in several galaxy types and use our models to predict molecular species that may be the best tracers of those conditions. Trust.

E.B. acknowledges financial support from the Leverhulme

\section{REFERENCES}

Aalto, S., Monje, R., \& Martín, S. 2007, A\&A, 475, 479

Aalto, S., Wilner, D., Spaans, M., Wiedner, M. C., Sakamoto, K., Black, J. H., \& Caldas, M. 2008, Ap\&SS, 313, 273

Baan, W. A., Henkel, C., Loenen, A. F., Baudry, A., \& Wiklind, T. 2008, A\&A, 477, 747

Bayet, E., Gerin, M., Phillips, T. G., \& Contursi, A. 2004, A\&A, 427, 45

Bayet, E., Gerin, M., Phillips, T. G., \& Contursi, A. 2006, A\&A, 460, 467

Bayet, E., Lintott, C., Viti, S., Martín-Pintado, J., Martín, S., Williams, D. A., \& Rawlings, J. M. C. 2008a, ApJ, 685, L35

Bayet, E., Viti, S., Williams, D. A., \& Rawlings, J. M. C. 2008b, ApJ, 676, 978

Bell, T. A., Roueff, E., Viti, S., \& Williams, D. A. 2006, MNRAS, 371, 1865

Bell, T. A., Viti, S., \& Williams, D. A. 2007, MNRAS, 378, 983

Boreiko, R. T., \& Betz, A. L. 1993, ApJ, 405, L39

Chieffi, A., \& Limongi, M. 2002, ApJ, 577, 281

Downes, D., \& Eckart, A. 2007, A\&A, 468, L57

Evans, A. S., Solomon, P. M., Tacconi, L. J., Vavilkin, T., \& Downes, D. 2006, AJ, 132, 2398
Ford, H. C., Dahari, O., Jacoby, G. H., Crane, P. C., \& Ciardullo, R. 1986, ApJ, 311, L7

Gao, Y., \& Solomon, P. M. 2004, ApJS, 152, 63

García-Burillo, S., et al. 2006, ApJ, 645, L17

Gerin, M., \& Phillips, T. G. 2000, ApJ, 537, 644

Gerin, M., \& Phillips, T. G. 2001, Ap\&SS, 277, 75

Graciá-Carpio, J., García-Burillo, S., Planesas, P., \& Colina, L. 2006, ApJ, 640, L135

Guélin, M., et al. 2007, A\&A, 462, L45

Habing, H. J. 1968, Bull. Astron. Inst. Netherlands, 19, 421

Heger, A., \& Woosley, S. E. 2002, ApJ, 567, 532

Imanishi, M., Nakanishi, K., Tamura, Y., Oi, N., \& Kohno, K. 2007, AJ, 134, 2366

Israel, F. P., \& Baas, F. 2001, A\&A, 371, 433

Israel, F. P., \& Baas, F. 2002, A\&A, 383, 82

Israel, F. P., \& Baas, F. 2003, A\&A, 404, 495

Israel, F. P., White, G. J., \& Baas, F. 1995, A\&A, 302, 343

Ivison, R. J., Smail, I., Bentz, M., Stevens, J. A., Menéndez-Delmestre, K., Chapman, S. C., \& Blain, A. W. 2005a, MNRAS, 362, 535

Ivison, R. J., et al. 2005b, MNRAS, 364, 1025

Ivison, R. J., et al. 2008, MNRAS, 390, 1117

Joy, M., Lester, D. F., Harvey, P. M., \& Frueh, M. 1986, ApJ, 307, 110

Knauth, D. C., Andersson, B.-G., McCandliss, S. R., \& Moos, H. W. 2003, ApJ, 596, L51

Knudsen, K. K., Walter, F., Weiss, A., Bolatto, A., Riechers, D. A., \& Menten, K. 2007, ApJ, 666, 156

Kohno, K., Matsushita, S., Vila Vilaró, B., Okumura, S. K., Shibatsuka, T., Okiura, M., Ishizuki, S., \& Kawabe, R. 2001, in ASP Conf. Ser. 249, The Central Kiloparsec of Starbursts and AGN: The La Palma Connection, ed. J. H. Knapen, J. E. Beckman, I. Shlosman, \& T. J. Mahoney (San Francisco, CA: ASP), 672

Kohno, K., Nakanishi, K., \& Imanishi, M. 2007, in ASP Conf. Ser. 373, The Central Engine of Active Galactic Nuclei, ed. L. C. Ho \& J.-W. Wang (San Francisco, CA: ASP), 647

Krips, M., Neri, R., García-Burillo, S., Martín, S., Combes, F., Graciá-Carpio, J., \& Eckart, A. 2008, ApJ, 677, 262

Krips, M., Peck, A. B., Sakamoto, K., Petitpas, G. B., Wilner, D. J., Matsushita, S., \& Iono, D. 2007, ApJ, 671, L5

Le Teuff, Y. H., Millar, T. J., \& Markwick, A. J. 2000, A\&AS, 146, 157

Lintott, C., \& Viti, S. 2006, ApJ, 646, L37

Lintott, C. J., Viti, S., Williams, D. A., Rawlings, J. M. C., \& Ferreras, I. 2005, MNRAS, 360, 1527

Martín, S., Martín-Pintado, J., Mauersberger, R., Henkel, C., \& García-Burillo, S. 2005, ApJ, 620, 210

Martín, S., Mauersberger, R., Martín-Pintado, J., Henkel, C., \& García-Burillo, S. 2006, ApJS, 164, 450

Meijerink, R., \& Spaans, M. 2005, A\&A, 436, 397

Meijerink, R., Spaans, M., \& Israel, F. P. 2007, A\&A, 461, 793

Meijerink, R., Tilanus, R. P. J., Dullemond, C. P., Israel, F. P., \& van der Werf, P. P. 2005, A\&A, 430, 427

Meyer, D. M., Jura, M., \& Cardelli, J. A. 1998, ApJ, 493, 222

Origlia, L., Ranalli, P., Comastri, A., \& Maiolino, R. 2004, ApJ, 606, 862

Pagani, L., Bacmann, A., Cabrit, S., \& Vastel, C. 2007, A\&A, 467, 179

Papadopoulos, P. P. 2007, ApJ, 656, 792

Pickles, J. B., \& Williams, D. A. 1981, Ap\&SS, 80, 337

Radford, S. J. E., Downes, D., \& Solomon, P. M. 1991, ApJ, 368, L15

Riechers, D. A., Walter, F., Carilli, C. L., \& Lewis, G. F. 2009, ApJ, 690, 463

Roberts, J. F., Rawlings, J. M. C., Viti, S., \& Williams, D. A. 2007, MNRAS, 382,733

Röllig, M., et al. 2007, A\&A, 467, 187

Sakamoto, K., et al. 2008, ApJ, 684, 957

Sembach, K. R., \& Savage, B. D. 1996, ApJ, 457, 211

Smith, L. J., Westmoquette, M. S., Gallagher, J. S., O’Connell, R. W., Rosario, D. J., \& de Grijs, R. 2006, MNRAS, 370, 513

Snow, T. P., Rachford, B. L., \& Figoski, L. 2002, ApJ, 573, 662

Sofia, U. J., Cardelli, J. A., Guerin, K. P., \& Meyer, D. M. 1997, ApJ, 482, L105

Soifer, B. T., et al. 1984, ApJ, 283, L1

Solomon, P. M., Downes, D., \& Radford, S. J. E. 1992, ApJ, 387, L55

Solomon, P. M., Vanden Bout, P. A., \& Maddalena, R. 2004, BAAS, 36, 824

Sosa-Brito, R. M., Tacconi-Garman, L. E., Lehnert, M. D., \& Gallimore, J. F. 2001, ApJS, 136, 61

Spaans, M., \& Meijerink, R. 2005, Ap\&SS, 295, 239

Umeda, H., \& Nomoto, K. 2002, ApJ, 565, 385 
van der Tak, F. F. S., Aalto, S., \& Meijerink, R. 2008, A\&A, 477, L5

Wagg, J., Wilner, D. J., Neri, R., Downes, D., \& Wiklind, T. 2005, ApJ, 634, L13

Weiß, A., Downes, D., Neri, R., Walter, F., Henkel, C., Wilner, D. J., Wagg, J., \& Wiklind, T. 2007, A\&A, 467, 955
Wu, J., Evans, N. J. II, Gao, Y., Solomon, P. M., Shirley, Y. L., \& Vanden Bout, P. A. 2005, ApJ, 635, L173

Younger, J. D., et al. 2008a, MNRAS, 387, 707

Younger, J. D., et al. 2008b, MNRAS, 387, 707

Zaritsky, D., Kennicutt, R. C., \& Huchra, J. P. 1994, ApJ, 420, 87 